\title{
Gastric amyloidosis: unusual cause of upper gastrointestinal hemorrhage
}
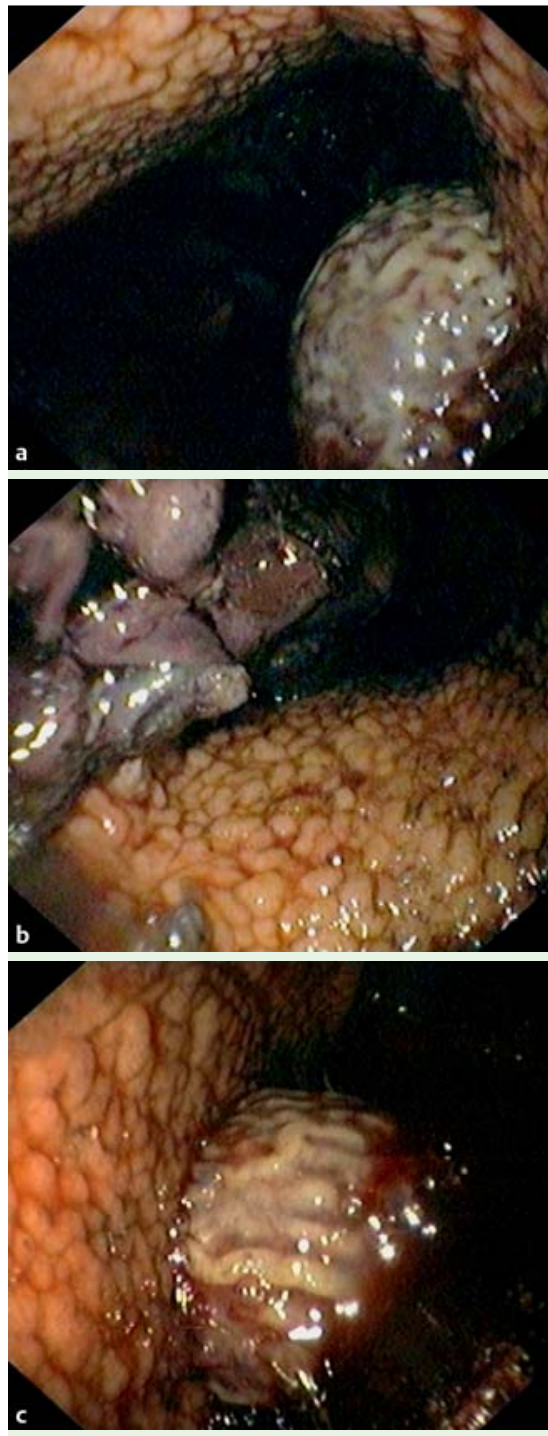

Fig. 1 a Gastric wall: endoscopic view of amyloidosis. b Areas of gastric mucosa infiltrated by whitish plaques. c Gastric mucosa infiltrated with oozing hemorrhage.

A 55-year-old man with multiple myeloma was admitted with hematemesis. After resuscitation, patient underwent endoscopy, which revealed a large vegetative mass covered by whitish plaques in the body/antrum transition of the greater curvature, with oozing bleeding ( $\bullet$ Fig. 1 ); the gastric walls were also covered by similar whitish lesions. Hemostasis with epinephrine injection was successful, and biopsy samples were taken. Pathology revealed chronic gastritis negative for Helicobacter pylori infection, fibrino-necrotic

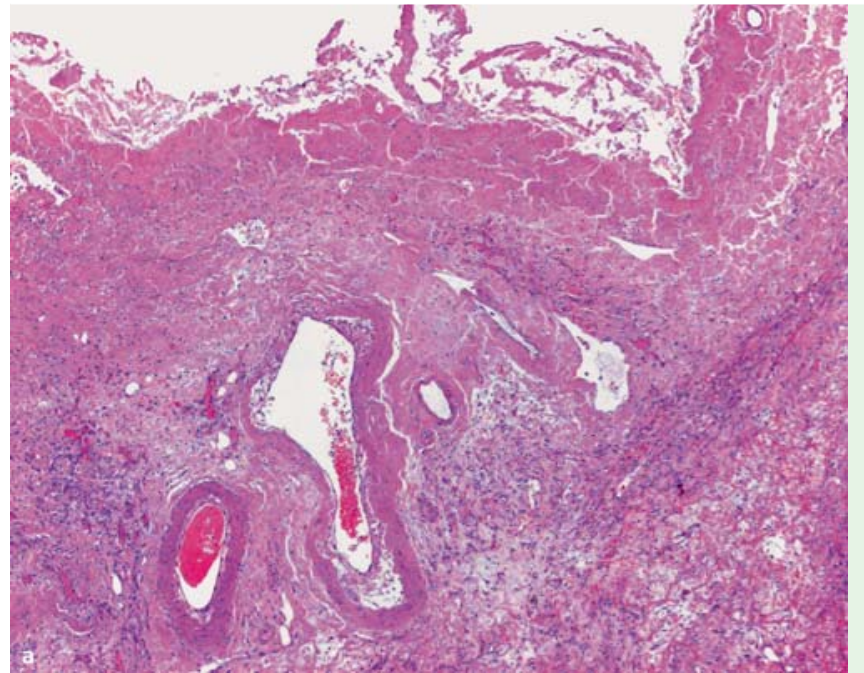

Fig. 2 a Histologic section (hematoxylin and eosin) showed fibrinonecrotic exudates, indicating ulceration of gastric mucosa. Note also the abundant deposits of eosinophilic and hyaline material. b Congo red staining showing deposits of eosinophilic and hyaline material positive for amyloid substance.

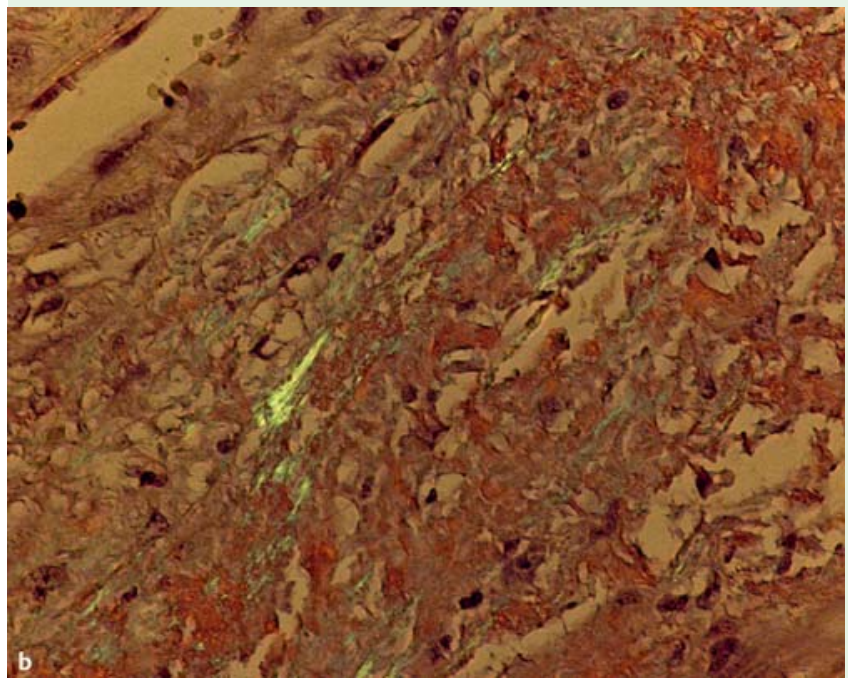

exudates, indicative of ulceration of gastric mucosa, and abundant eosinophilic and hyaline deposits which were positive for amyloid substance ( $\bullet$ Fig. 2 ).

The patient re-presented with further episodes of hematemesis and due to lack of effective endoscopic hemostasis, gastrectomy was carried out. The surgical specimen showed extensive ulceration of gastric mucosa, associated with amyloid deposits, without evidence of euplastic involvement. The patient died 1 week later with infectious complications.

Amyloid deposits are produced in a variety of diseases and may be present in one or multiple organs [1]. Primary amyloidosis is associated with monoclonal light chains in the serum and/or urine with $15 \%$ of patients having multiple myeloma.
Secondary amyloidosis is associated with inflammatory, infectious, and neoplastic diseases. The gastrointestinal tract is one of the most commonly involved regions in systemic amyloidosis, although it rarely affects the stomach [2]. Isolated amyloidosis in the stomach is even more rare [3]. Amyloid-related gastric symptoms were reported in $1 \%$ of a large series of systemic amyloidosis presenting as: tumorlike growth, erosive lesions with hemorrhages, outlet obstruction, and gastroparesis with autonomic failure [4]. Gastrointestinal bleeding is a rare initial symptom, albeit fatal in some cases [5]. No specific treatment is available for the gastrointestinal complications of amyloidosis. The role of endoscopy in amyloidosis-related hemorrhage is limited. 
Endoscopy_UCTN_Code_CCL_1AB_2AD_3AF

\section{Competing interests: None}

M. Marques ${ }^{1,2}$, J. A. Sarmento ${ }^{1,2}$,

S. Rodrigues ${ }^{1}$, S. Guimarães ${ }^{3}$,

E. Fonseca ${ }^{3}$, G. Macedo ${ }^{1,2}$

1 Gastroenterology Department, Hospital São João, Porto, Portugal

2 Faculty of Medicine, University of Porto, Porto, Portugal

3 Pathology Department, Hospital São João, Porto, Portugal

\section{References}

1 Dias VC, Tavares I, Gonçalves R, Macedo G. AL-amyloidosis presenting as massive gastrointestinal bleeding. Am J Gastroenterol 2009; 104: 2374-2376

2 Wu D, Lou JY, Chen J et al. A case report of localized gastric amyloidosis. World J Gastrenterol 2003; 9: 2632 - 2634

3 Losanoff JE, Antaki F, Salwen WA et al. Amyloid tumor of the stomach simulating an obstructing carcinoma: case report and review of the literature. Endoscopy 2009; 41 (Suppl 2): E45-E46

4 Iijima-Dohi N, Shinji A, Shimizu T et al. Recurrent gastric hemorrhaging with large submucosal hematomas in a patient with primary AL systemic amyloidosis: endoscopic and histopathological findings. Intern Med 2004; 43: $468-472$

$5 \mathrm{Kim}$ H, Jee SR, Lee SB et al. A case of secondary amyloidosis presenting as massive gastrointestinal bleeding. Korean J Gastroenterol 2006; 47: 397-401
Bibliography

DOI $10.1055 / \mathrm{s}-0030-1256080$

Endoscopy 2011; 43: E100 -E101

(c) Georg Thieme Verlag KG Stuttgart · New York . ISSN 0013-726X

\section{Corresponding author}

\section{Dr. M. Marques}

Hospital São João - Alameda Hernani Monteiro 4200 Porto

Portugal

Fax: $+351-225-512100$

mgmarques@net.sapo.pt 